\title{
Variations in EUV Irradiance: Comparison between LYRA, ESP, and SWAP Integrated Flux
}

\author{
Mehmet Sarp Yalim and Stefaan Poedts \\ Center for Mathematical Plasma Astrophysics, Department of Mathematics, KU Leuven, Campus Arenberg, \\ Celestijnenlaan 200b, Bus 2400, 3001 Leuven, Belgium
}

Correspondence should be addressed to Mehmet Sarp Yalim; yalimsarp@hotmail.com

Received 25 November 2013; Revised 20 March 2014; Accepted 4 April 2014; Published 9 June 2014

Academic Editor: Dean Hines

Copyright (C) 2014 M. S. Yalim and S. Poedts. This is an open access article distributed under the Creative Commons Attribution License, which permits unrestricted use, distribution, and reproduction in any medium, provided the original work is properly cited.

\begin{abstract}
The Sun Watcher Using Active Pixel System Detector and Image Processing (SWAP) telescope and Large Yield Radiometer (LYRA) are the two Sun observation instruments on-board PROBA2. SWAP extreme ultraviolet images, if presented in terms of the integrated flux over solar disk, in general, correlate well with LYRA channel 2-4 (zirconium filter) and channels QD and 18 of EVE/ESP on-board SDO between 2010 and 2013. Hence, SWAP can be considered as an additional radiometric channel. We compare in detail LYRA channel 2-4 and SWAP integrated flux in July 2010 and in particular during the solar eclipse that occurred on July 11, 2010. During this eclipse, the discrepancy between the two data channels can be explained to be related to the occultation of active region 11087 by the Moon. In the second half of July 2010, LYRA channel 2-4 and SWAP integrated flux deviate from each other, but these differences can also be explained in terms of features appearing on the solar disk such as coronal holes and active regions. By additionally comparing with timeline of EVE/ESP, we can preliminarily interpret these differences in terms of the difference between the broad bandpass of LYRA channel 2-4 and the, relatively speaking, narrower bandpass of SWAP.
\end{abstract}

\section{Introduction}

ESA's Project for On-Board Autonomy 2 (PROBA2) mission [1-3] was launched on November 2, 2009 from Plesetsk, Russia, with a Rockot launcher to a Sun-synchronous orbit at an altitude of $725 \mathrm{~km}$. It provides a technology demonstration platform for testing a number of instruments and techniques relevant to solar physics, space weather, aeronomy, avionics, spacecraft attitude control, power system, and propulsion. The orbit of PROBA2 is eclipse-free for nine months every year. It tracks the terminator, which is the dividing line between day and night on Earth, over the poles, however, not exactly. As a result, during approximately $80 \mathrm{~d}$ from November until January every year, PROBA2 experiences eclipses of the Sun by the Earth every orbit with a duration of maximum $25 \mathrm{~min}$, where an orbit is about $100 \mathrm{~min}$ long [4]. In general, the orbit is well suited for continuous solar observations by the primary scientific instruments [5] on-board: the Sun Watcher Using Active Pixel System Detector and Image Processing (SWAP) and the Large Yield Radiometer (LYRA).

SWAP [6-9] is an extreme ultraviolet (EUV) full-Sun imager that observes the Sun in a narrow bandpass with peak at $17.4 \mathrm{~nm}$ [9]. Its bandpass covers some of the brightest spectral lines of iron ions emitted by the solar corona, Fe IXXI, with maximum excitation temperatures $\left(\lg T_{\max }=5.9\right.$ to 6.1) [6], and contains a small number of chromospheric and photospheric lines.

SWAP was built on the heritage of the Extreme Ultraviolet Imaging Telescope (EIT) [10] on-board the ESA/NASA joint mission Solar and Heliospheric Observatory (SOHO). It continues EIT's systematic CME watch program at an improved average cadence of $1 \mathrm{~min}$ to $2 \mathrm{~min}$ to better monitor events in the low solar corona that might be relevant for space weather. Furthermore, SWAP has a larger field-of-view (FOV) than EIT (54 arcmin versus 45 arcmin), and it has the capability 
of off-pointing to further track the evolution of off-limb solar ejecta.

The NASA Solar Dynamics Observatory (SDO) mission is a much larger mission than PROBA2 and the Atmospheric Imaging Assembly (AIA) [11] is better than SWAP in every way, except that the FOV of SWAP is larger than the FOV of AIA, viz. 41 arcmin, and moreover, SWAP has the capability of off-pointing.

LYRA $[4,12,13]$ is a vacuum ultraviolet (VUV) solar radiometer. It monitors the solar irradiance in four UV passbands which were chosen for their relevance to solar physics, aeronomy, and space weather. These are (1) the (120 to 123) nm Lyman-alpha channel including the H I $121.6 \mathrm{~nm}$ Lymanalpha line; (2) the (190 to 222) nm Herzberg continuum range; (3) the (17 to 80$) \mathrm{nm}$ aluminum filter channel including the He II $30.4 \mathrm{~nm}$ line as well as soft X-ray (SXR) radiation below $5 \mathrm{~nm}$; (4) the (6 to 20) nm zirconium filter channel including SXR radiation below $2 \mathrm{~nm}$, where the solar variability is the highest. LYRA consists of three identical units each of which has all the four channels. Each of these units is used with a different utilization frequency enabling the assessment of the evolution of radiometric sensors and filters. In this paper, we will focus on the measurements made by unit 2 which is the nominal unit in permanent use and utilizes diamond detectors for all its channels [4].

SWAP and LYRA operate side-by-side on-board PROBA2. Together, they establish a high-performance solar monitor for operational space weather nowcasting and research. In this paper, we will demonstrate the capability of SWAP to be used as an additional radiometric channel. We make a different analysis than that presented in [14]. Accordingly, we will first present the solar irradiance measurements obtained by LYRA channel $2-4$, the channels QD ((0.1 to 7) $\mathrm{nm})$ and 18 ((17.5 to 21.1) nm) of the Extreme Ultraviolet Variability Experiment/Extreme Ultraviolet Spectrophotometer (EVE/ESP) onboard SDO, and the SWAP integrated flux between 2010 and 2013. Afterwards, we will focus on a shorter period and discuss the solar irradiance variation of two particular active regions, viz. 11087 and 11089, which dominated the Earthfacing solar disk in July 2010. Finally, particular emphasis will be given to the SWAP and LYRA observations made during the July 11, 2010 solar eclipse and irradiance variation during the occultation of the active region 11087 by the Moon. The observation data will be supported by the SWAP and AIA integrated fluxes computed by simulating the Moon transits over the solar disk in noneclipse level 1 images taken on July 11,2010 by both the SWAP $(17.4 \mathrm{~nm})$ and the AIA $9.4 \mathrm{~nm}$ and $13.1 \mathrm{~nm}$ channels.

The paper is structured as follows. In Section 2, we present some specifications of the utilized SWAP and LYRA data; in Section 3, we present our results and discuss them. In particular, in Section 3.1, we present our results on the trend comparison between the SWAP integrated flux, LYRA, and ESP data between 2010 and 2013, and also focus on July 2010; and in Section 3.2, we focus on the results during the July 11, 2010 eclipse. Finally, in Section 4, we present our conclusions.

\section{Data Specifications}

2.1. SWAP Average Intensity. SWAP average intensity (SWAVINT) is a keyword in the calibrated (i.e., level 1) SWAP FITS files and corresponds to the average intensity of a calibrated SWAP image $[9,14]$. The calibration steps used involve bad or hypersensitive pixel corrections, missing, saturated or negative-valued pixel corrections, dark current subtraction, flat-field correction, despiking, image transformations, and exposure time normalization. Only the last step of the default calibration process, the centering of the solar disk in the image and rotation to Solar North, is applied after calculating SWAVINT. Consider the following:

$$
\text { SWAVINT }=\frac{1}{t} \frac{1}{P} \sum_{p=1}^{P} \mathrm{DN}_{p}
$$

where $t$ denotes the exposure time of the image, $P$ is the number of pixels which is $1024 \times 1024$ for the whole image, and $\mathrm{DN}_{p}$ denotes the digital number in pixel $p$ after calibration [14]. The unit of SWAVINT is DN. $\mathrm{s}^{-1}$.

The detector response of SWAP has been analyzed in detail in [8]. Accordingly, by monitoring the in-flight detector response of SWAP using two current-driven near-UV LEDs that emit at $500 \mathrm{~nm}$ and additionally by comparing SWAVINT with the synthetic response generated from the calibrated level 2 (version 2) spectra of EVE during the first two years of the SWAP mission, it was concluded in [8] that SWAP has not significantly degraded during the considered period of time.

SWAVINT can be regarded as a solar EUV irradiance at the SWAP bandpass centered at $17.4 \mathrm{~nm}$. The dynamic range of SWAP, which is 12 bit, is limited to see flares at full extent. Consequently, the few SWAP pixels building up the flare site are soon saturated and so the flares can hardly be seen in SWAVINT [14]. However, as will be demonstrated in Section 3.1, the variations in SWAVINT which do not contain the short-term spiky SXR response have a very good agreement with the long-term background EUV response of the LYRA channel 2-4 data and therefore can, in principle, be used to separate the SXR and EUV components of the LYRA channel 2-4 data.

2.2. LYRA Data. In this paper, two types of LYRA data products [4] are used.

For long-term variations of LYRA channel 2-4 (e.g., monthly), which will be of interest in Section 3.1, the $1 \mathrm{~min}$ averaged calibrated LYRA data (i.e., level 3) are used. This data product is based on the base science data (i.e., level 2). These signals are cleaned for temperature and degradation effects, normalized by exposure time, and calibrated to $\mathrm{W} \cdot \mathrm{m}^{-2}$.

For short-term variations (e.g., during the July 11, 2010 solar eclipse) which will be focused on in Section 3.2, the full standard raw LYRA data (i.e., level 1), having the unit of $\mathrm{kHz}$ and obtained with a cadence of $50 \mathrm{~ms}$, are utilized without applying any averaging. As will be demonstrated in Section 3.2, the LYRA unit 2 signals make dips during 


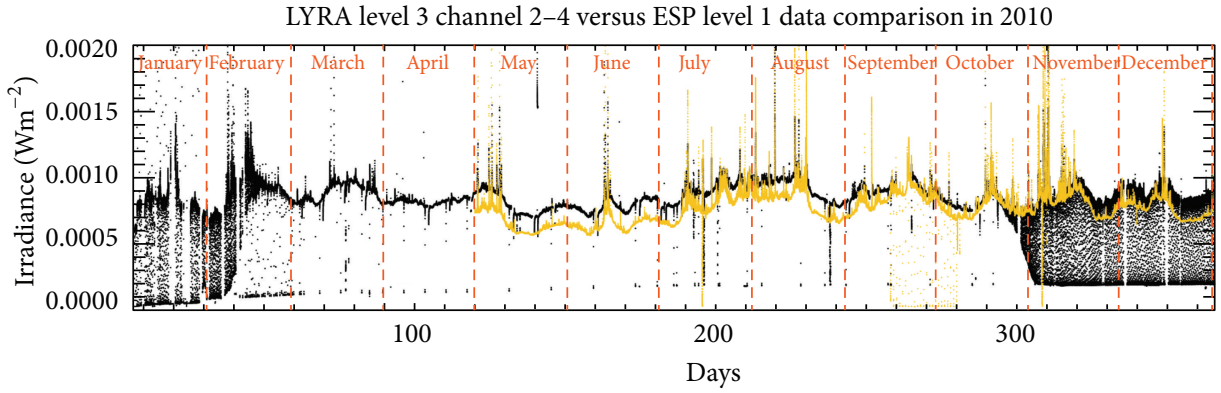

* LYRA level 3 channel 2-4 data

* ESP level 1 data (combined response of channels QD and 18)

(a)

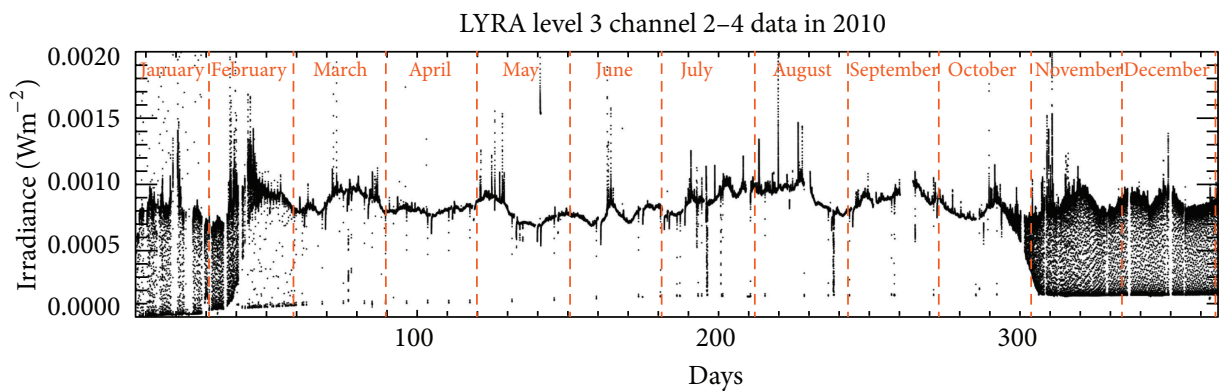

(b)

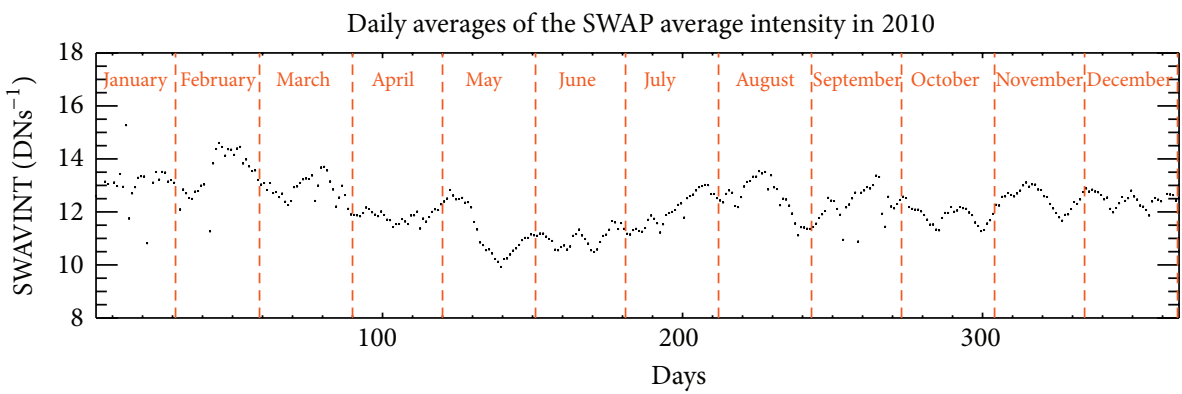

(c)

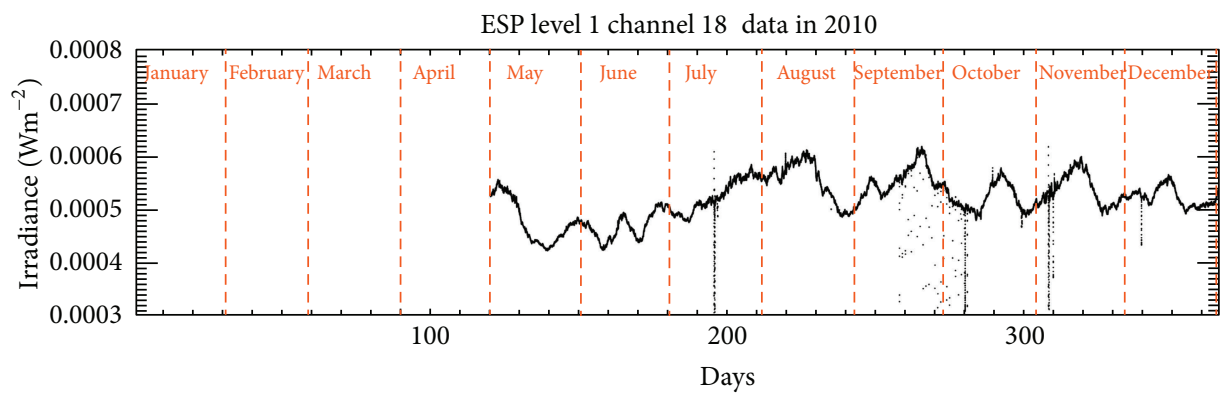

(d)

Figure 1: From top to bottom: LYRA level 3 channel 2-4 and ESP level 1 data (at combined responses of the channels QD and 18); LYRA channel 2-4 data alone; daily averages of SWAVINT data; and ESP channel 18 data variations in 2010.

the solar eclipse. The minimum levels of these dips are systematically overestimated since the correction to the degradation effects, which were considerably present in unit 2 of LYRA at the time of the solar eclipse, is additive. Therefore, we present the LYRA unit 2 eclipse measurements with the level 1 data product.

\section{Results and Discussion}

3.1. Trend Comparison and Influence of Coronal Features. In Figure 1, the two upper figures show the LYRA level 3 channel 2-4 response in 2010. This demonstrates the variation of the $\mathrm{X}$ and EUV corona based on the LYRA channel 
2-4 response, with upward spikes being associated with solar flares. However, not all variations shown in the plot are of solar origin. The frequent drops in signal observed in the periods of January 6, 2010 to February 22, 2010 and from 15 October 2010 onwards are caused by PROBA2's eclipse season. During this period, the LYRA signals, including the zirconium signal, are (partially) absorbed once per orbit during a time period which ranges from a few minutes to about $1 \mathrm{~h}$. Also, during spacecraft off-pointing campaigns, LYRA suffers from a drop in signal as the Sun moves (partially) out of its FOV, viz. by 2 arcdeg. The small data gaps are a consequence of calibration campaigns or times at which LYRA was temporarily switched off. These gaps are always followed by a drop in signal strength which slowly increases to the true solar irradiance level as the diamond detectors need time to stabilize.

The uppermost figure shows also the $1 \mathrm{~min}$ averaged calibrated ESP level 1 data variation in 2010 at combined responses of its channels QD ((0.1 to 7$) \mathrm{nm})$ and 18 ((17.5 to 21.1) $\mathrm{nm}$ ). These combined responses correlate well with the LYRA channel 2-4 response. However, there are differences in signal values due to the differences in the bandpasses of LYRA and ESP channels.

The third figure presents the SWAVINT variation of the solar corona at $17.4 \mathrm{~nm}$ in 2010 based on SWAP level 1 images. The low value black dots in July 2010 correspond to the July 11, 2010 solar eclipse which will be discussed in detail in Section 3.2.

The lowest figure shows the ESP channel 18 data variation in 2010 which is in good correlation with the SWAVINT variation. These two datasets demonstrate the variations of the EUV corona on 17.5 to $21.1 \mathrm{~nm}$ and $17.4 \mathrm{~nm}$ bandpasses, respectively.

The LYRA channel 2-4 and SWAVINT variations show a good correlation at first glance. On top of the EUV variation seen in both signals, the LYRA channel 2-4 shows the SXR and EUV power of solar flares.

Figures 2, 3, and 4 are similar to Figure 1; but the data variations correspond to 2011, 2012, and 2013, respectively. The aforementioned correlations between the LYRA, ESP, and SWAVINT data are valid between 2011 and 2013 as well. In addition, the measured irradiance levels gradually increase for all channels (especially SWAVINT and ESP channel 18) in 2011. In 2012 and 2013, SWAVINT, ESP channel 18, and combined ESP channel measurements indicate higher average irradiance values in comparison with their average values in 2010

In Figure 5, the top figure shows the variation in LYRA channel 2-4 signal in July 2010. The grey bands denote periods during which calibration campaigns were held and scientific data are missing. The orange periods denote special campaigns in the Sun but pointing slightly away from the Sun center. This can have both positive and negative effects on the LYRA signals as it depends on the flatfield of the detectors. The other downward spikes are due to the stabilization effect after the spacecraft off-pointings required by SWAP calibration campaigns.

The bottom figure presents the SWAVINT variation in July 2010. The effect of the solar eclipse on July 11, 2010 is clearly seen as a significant drop in signal due to the occultation of the solar disk by the Moon.

The SWAP and LYRA channel 2-4 irradiances in July 2010 have similar trends but there is a clear difference. The LYRA curve displays two distinct "bumps" starting at the time when the active regions 11087 and 11089 appeared on disk, viz. after July 6, 2010 and July 16, 2010, respectively. The same behavior is observed by the ESP level 1 data corresponding to the combined responses of the channels QD and 18 (see Figure 6). In general, LYRA channel 2-4 and ESP level 1 data at combined responses of the channels QD and 18 are in good qualitative agreement. Furthermore, there is a good correlation between SWAVINT and ESP channel 18 data in July 2010 (see Figure 6). Thus, this supports our main point in this paper that SWAVINT can be utilized as an additional radiometric channel.

The second "bump" that occurs after July 16, 2010 in the LYRA channel 2-4 data is not seen in the SWAVINT variation. A possible explanation for this is the compensation of competing effects like active regions and coronal holes. In order to demonstrate this, SWAP average intensities of the northern and southern solar disk of the Sun are computed separately by applying the same formula as in (1) to the pixels that have a coronal signal related to the northern and southern solar disk, respectively, instead of the whole image. The results are shown in Figure 7 where, in general, the spurious data points correspond to campaign data. It can be deduced that around the time of the appearance of the second "bump" in the LYRA channel 2-4 data, a large coronal hole appeared in the northern solar disk while the active region 11089 was passing in the southern solar disk. Thus, they compensate each other. It should be noted that LYRA channel 2-4 is more biased towards the active regions due to its SXR component. Therefore, a "bump" is still observable in the LYRA channel 2-4 data in spite of the compensation effect. However, for SWAP, the effects of the background solar irradiance can be as significant as the irradiance from the active regions.

3.2. Analysis of the July 11, 2010 Solar Eclipse. Having compared the SWAVINT and LYRA channel 2-4 variations in July 2010 and analyzed the major differences based on solar features in Section 3.1, in this section, our main aim is to analyze the effects of the July 11, 2010 solar eclipse on the LYRA channels and SWAVINT. However, since a significant number of the SWAP images taken during the solar eclipse correspond to special observation campaigns, the SWAVINT trend is mostly not clear enough. As a remedy, a simulated SWAVINT variation based on artificial eclipse images is computed by using (1). These images are created with $10 \mathrm{~s}$ cadence from the noneclipse SWAP level 1 image taken at 2010-0711T17:36:07Z UTC by means of occulting the solar disk by the Moon, whose position with respect to the solar disk in a SWAP image is predicted by the Pointing, Positioning and Time (PPT) software module of the PROBA2 Science Center (P2SC) [15]. We refer the reader to the Supplementary Material available online at http://dx.doi.org/10.1155/2014/957461 to watch the movie consisting of these images. 


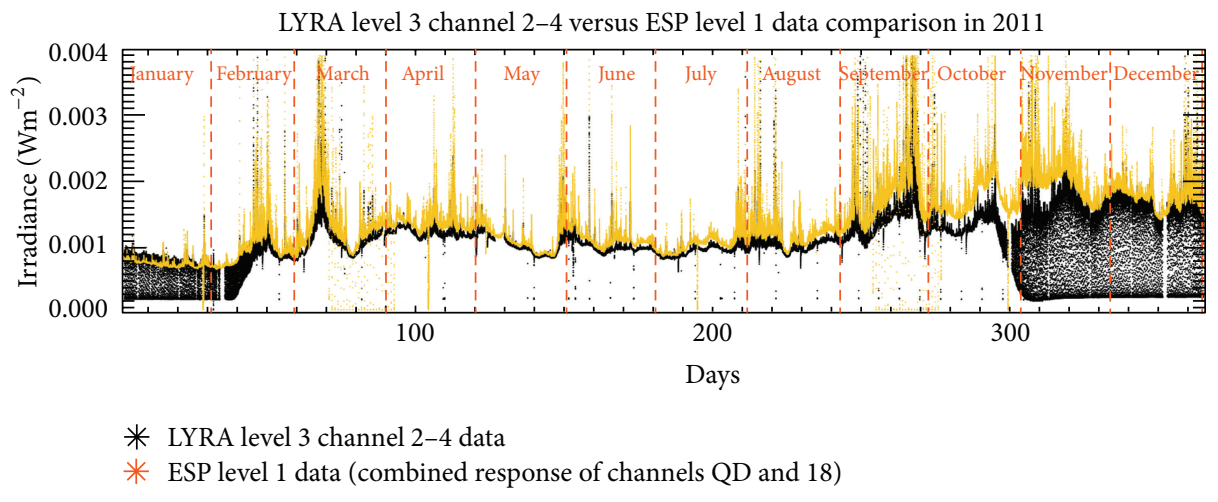

(a)

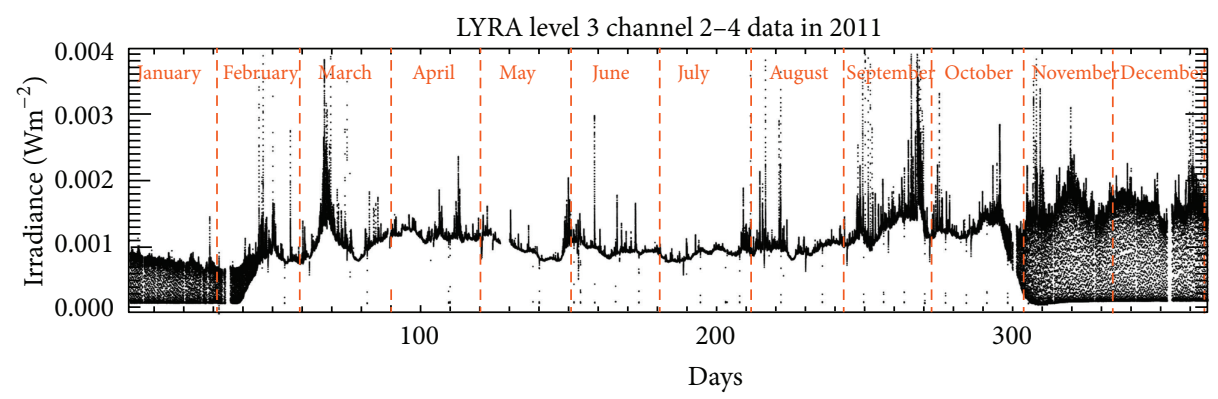

(b)

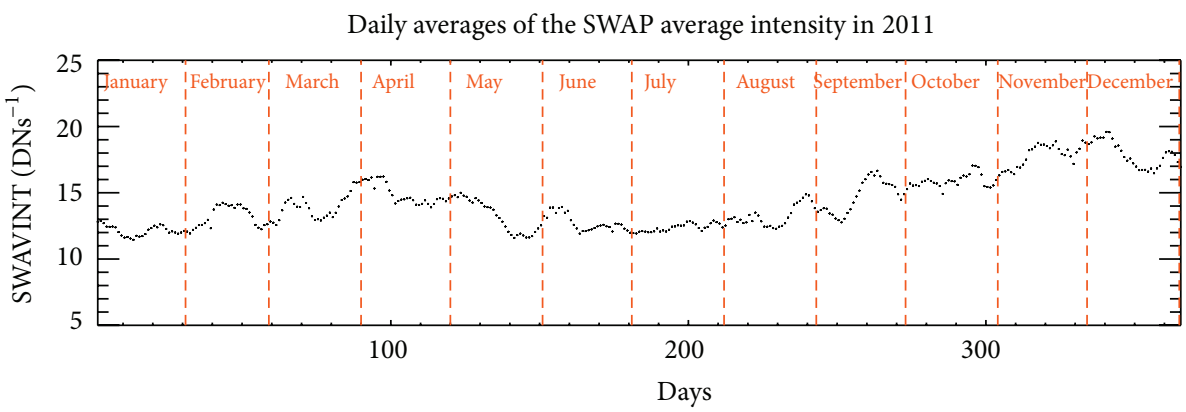

(c)

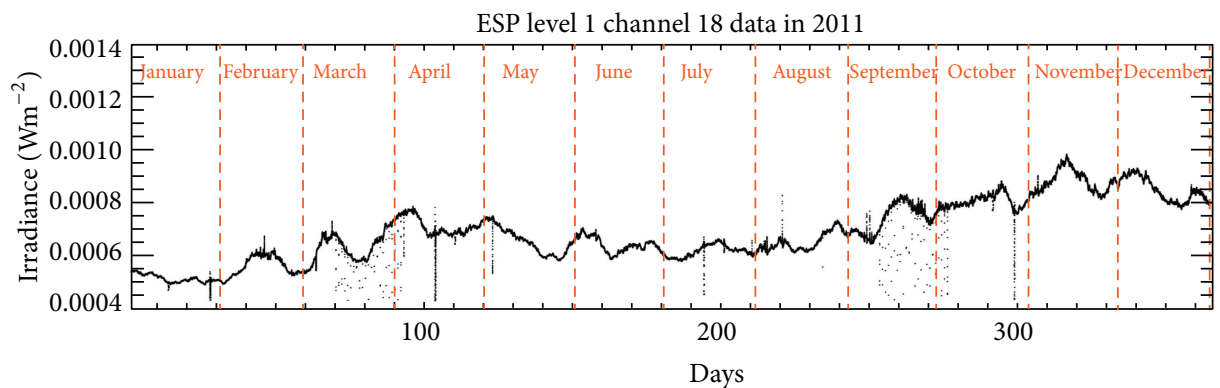

(d)

FIgURE 2: From top to bottom: LYRA level 3 channel 2-4 and ESP level 1 data (at combined responses of the channels QD and 18); LYRA channel 2-4 data alone; daily averages of SWAVINT data; and ESP channel 18 data variations in 2011. 


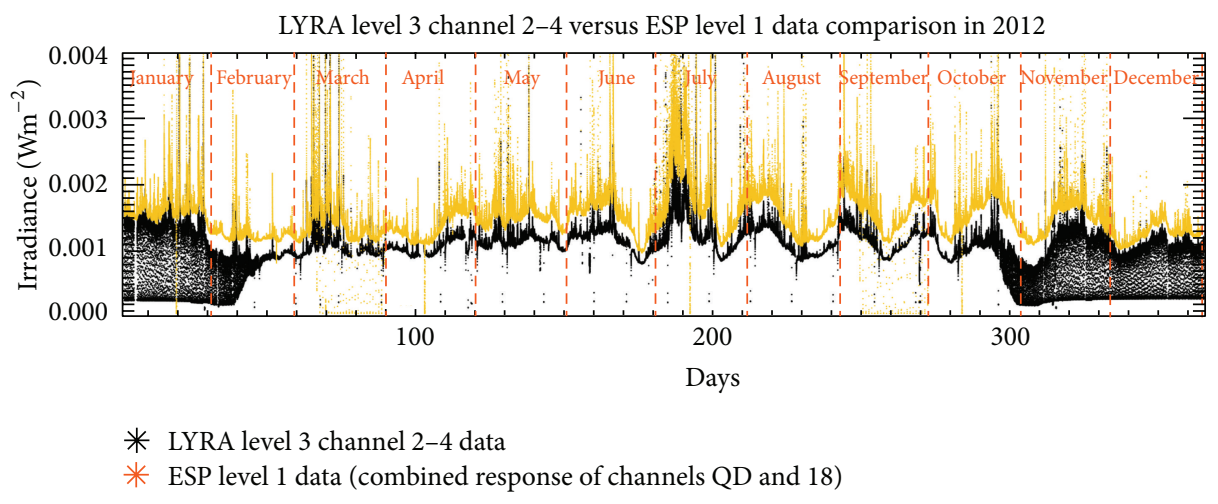

(a)

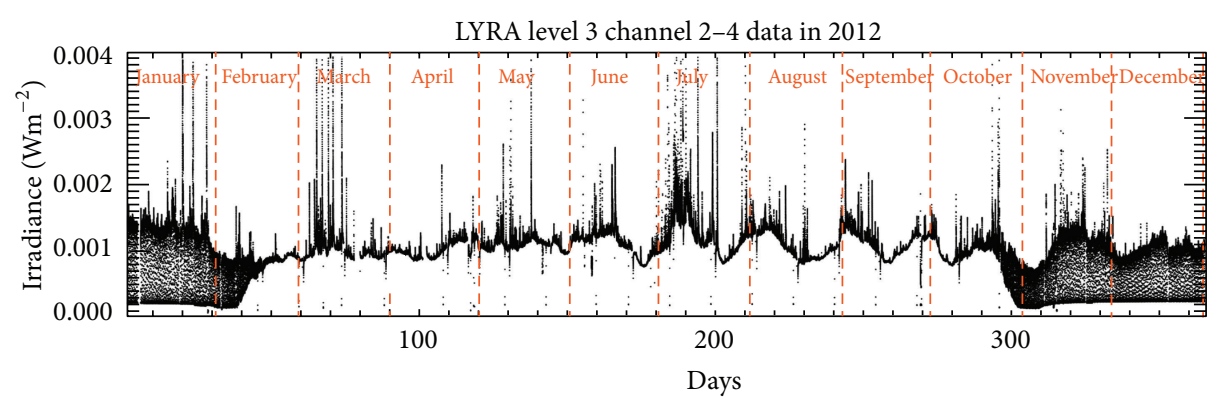

(b)

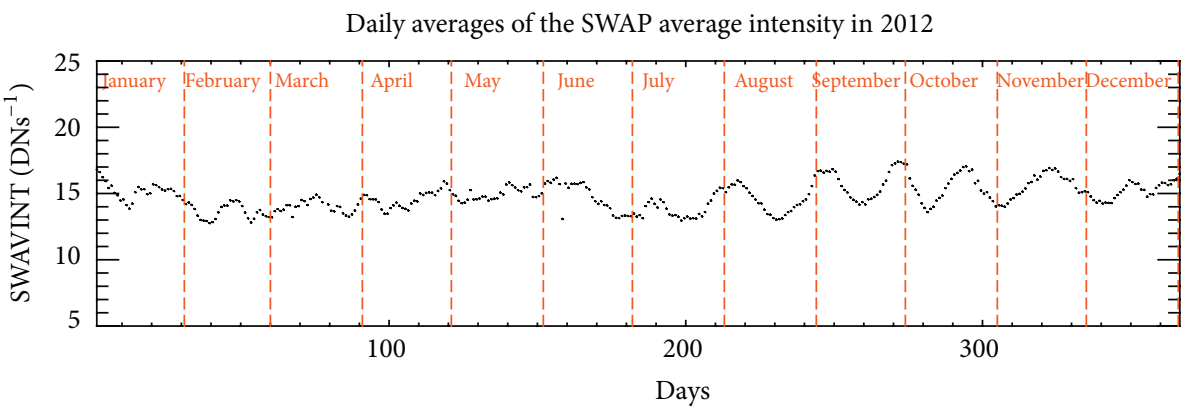

(c)

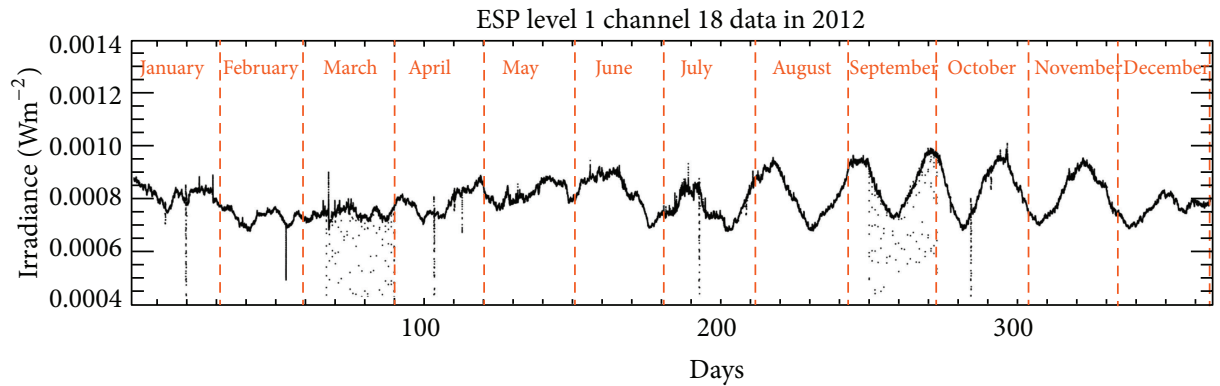

(d)

FIGURE 3: From top to bottom: LYRA level 3 channel 2-4 and ESP level 1 data (at combined responses of the channels QD and 18); LYRA channel 2-4 data alone; daily averages of SWAVINT data; and ESP channel 18 data variations in 2012. 


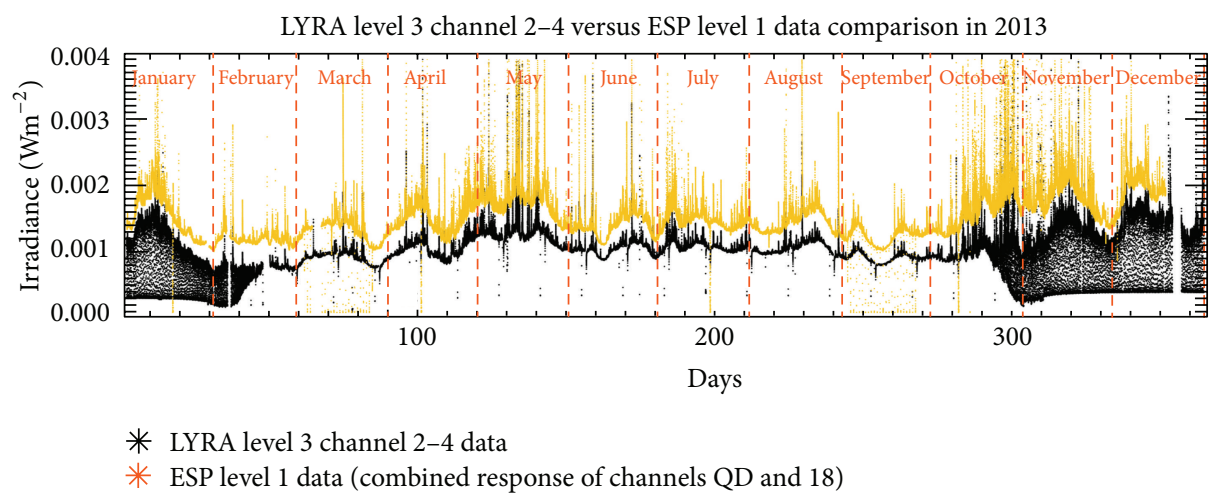

(a)

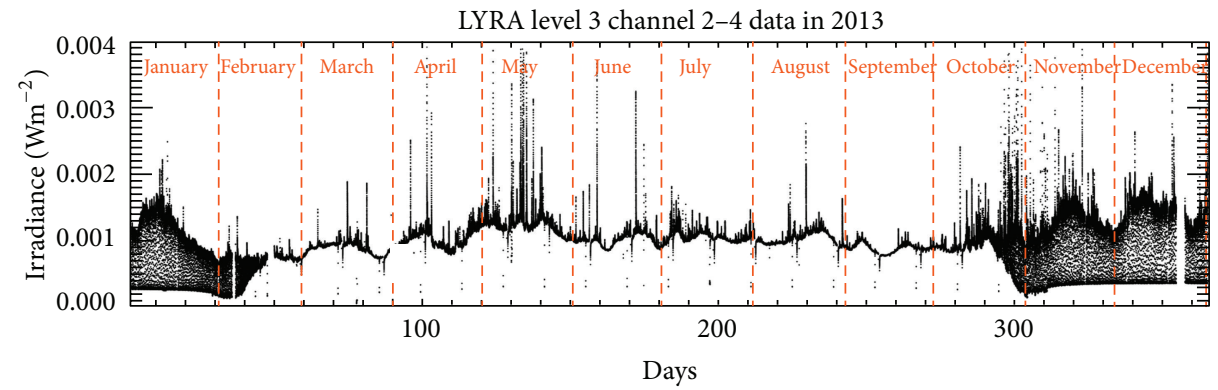

(b)

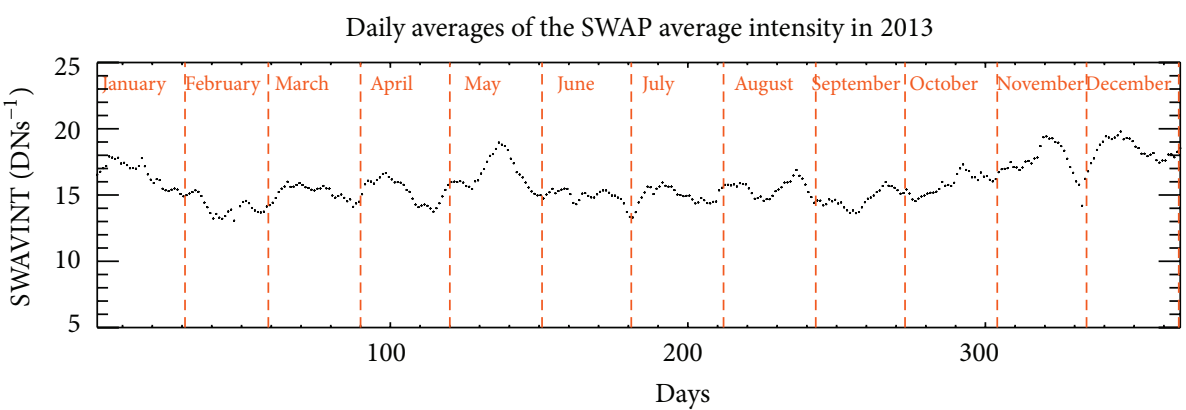

(c)

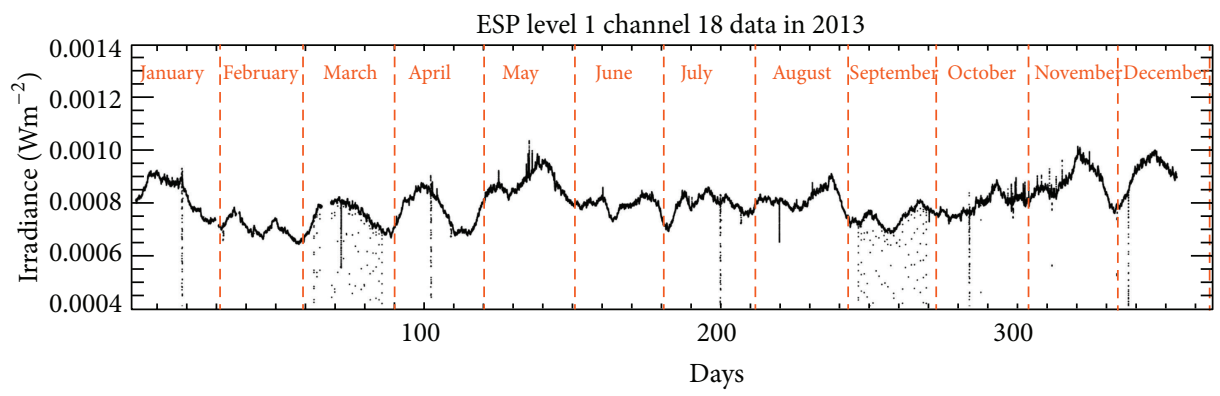

(d)

FIgURE 4: From top to bottom: LYRA level 3 channel 2-4 and ESP level 1 data (at combined responses of the channels QD and 18); LYRA channel 2-4 data alone; daily averages of SWAVINT data; and ESP channel 18 data variations in 2013. 


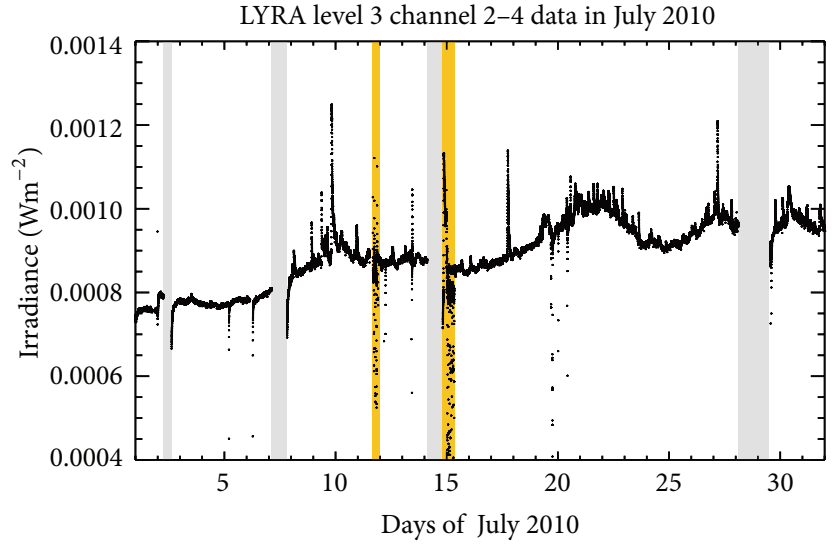

(a)

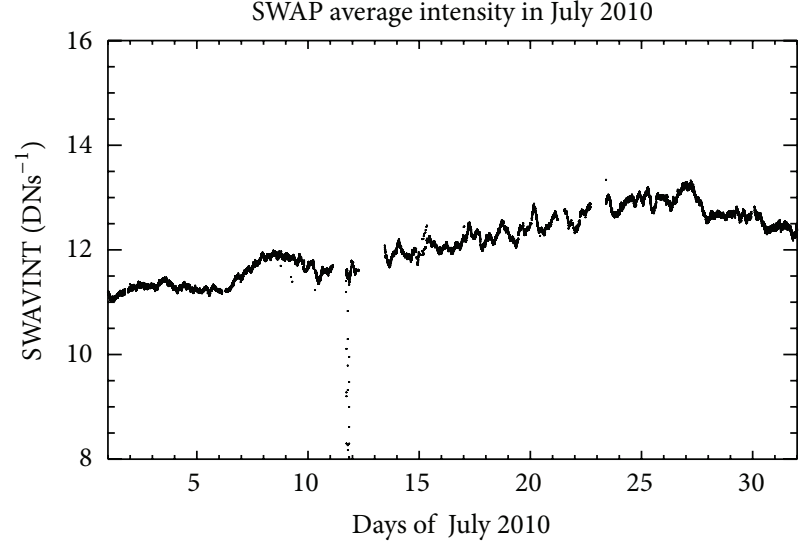

(b)

FIGURE 5: SWAVINT data variation (b) and LYRA level 3 channel 2-4 data variation (a) in July 2010 with calibration campaign data (grey bands) and special campaign data (orange bands) indicated. The other downward spikes are a result of the stabilization effect after the spacecraft off-pointings required by the SWAP calibration campaigns.
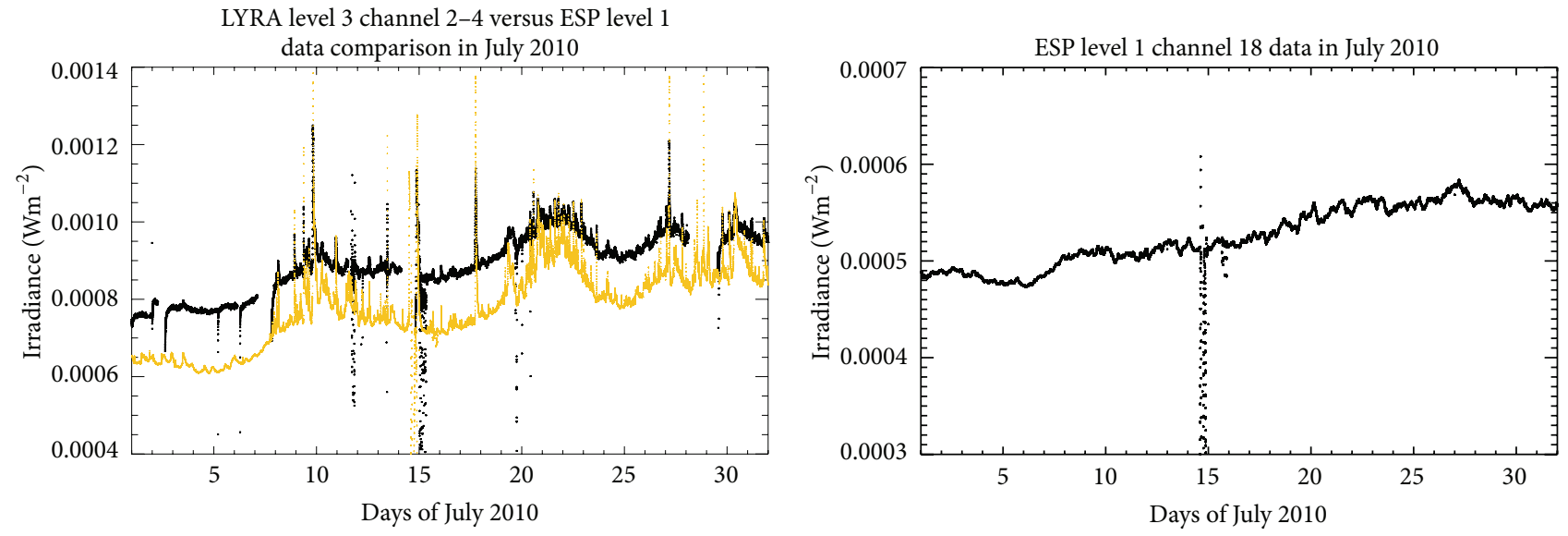

* LYRA level 3 channel 2-4 data

ESP level 1 data (combined response of channels QD and18)

(a)

(b)

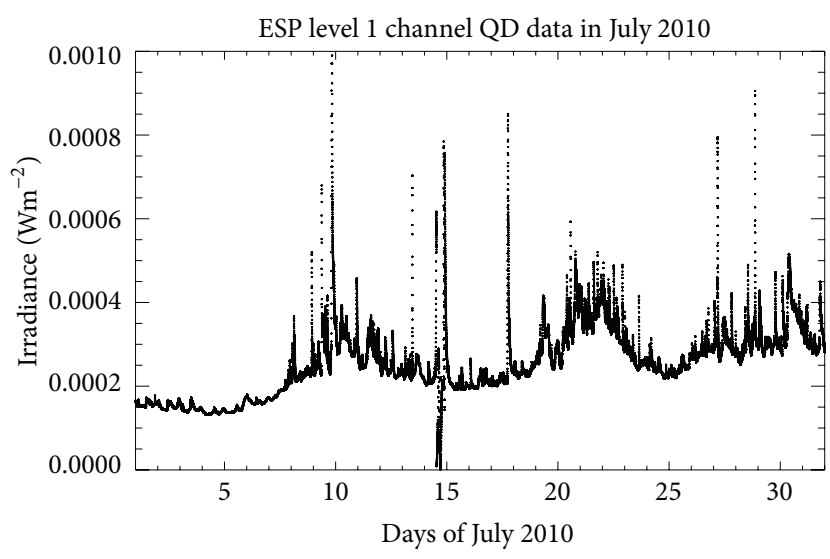

(c)

FIGURE 6: LYRA level 3 channel 2-4 and ESP level 1 data variations (at combined responses of the channels QD and 18, and separately) in July 2010. The downward spikes in the ESP data on July 14, 2010 are due to the calibration maneuvers of SDO. 


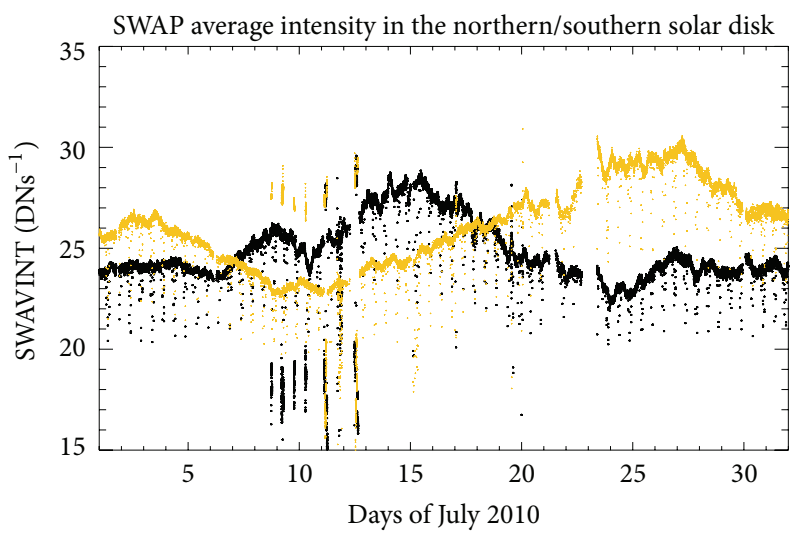

* Northern disk

Southern disk

FIGURE 7: SWAP average intensity variations in the northern and southern solar disk in July 2010.

In order to see the variation at different wavelengths in the LYRA channel 2-4 bandpass other than the SWAP bandpass, average intensities corresponding to the artificial eclipse images created from the AIA $9.4 \mathrm{~nm}$ and $13.1 \mathrm{~nm}$ level 1 images taken at 2010-07-11T18:59:32Z UTC and 2010-0711T18:59:33Z UTC, respectively, are computed following the same procedure applied for SWAP and by using the same formula given in (1).

While the active region 11087 was situated on the northeast disk, PROBA2 experienced a solar eclipse on July 11, 2010. The July 11, 2010 solar eclipse was seen as a total eclipse on Earth with the partial eclipse begin and end times as 2010-07-11T17:09:37Z UTC and 2010-07-11T21:57:14Z UTC, respectively, and the total eclipse begin and end times as 2010-07-11T18:15:12Z UTC and 2010-07-11T20:51:41Z UTC, respectively (see http://eclipse.gsfc.nasa.gov/SEmono/TSE2010/TSE2010fig/TSE2010-fig301.pdf). On the other hand, PROBA2 saw it as four partial eclipses due to the characteristics of its orbit.

In Figures 8, 9, and 10, LYRA level 1 data measured by all four channels of unit 2 (i.e., Lyman-alpha, Herzberg continuum, aluminum, and zirconium) and SWAVINT variations are presented during the first three occultations (the fourth occultation is similar to the first one for our purpose, so we do not show it here) along with the simulated SWAVINT and AIA average intensity variations.

All four LYRA channels show a decrease in irradiance during these occultations, but the short-wavelength channels 2-3 and 2-4 show asymmetries and additional dips when the Moon covered the active region 11087 during the second and third transits. This is due to the blockage of the SXR component of the irradiance coming from the active region.

The observed and simulated SWAVINT variations as well as the simulated AIA average intensity variations at $9.4 \mathrm{~nm}$ and $13.1 \mathrm{~nm}$ show a similar, symmetric decrease-increase profile during the first transit where the active region 11087 was not occulted.

During the second transit, all four average intensity variations show an asymmetric profile with a second dip when the active region 11087 was occulted similar to the LYRA short-wavelength channels. However, during the third transit, although the four variations have an asymmetric profile, they show a bump instead of a dip when the active region 11087 was occulted. The reason for this main difference between the profiles obtained during the second and third transits is related to the way of occultation of the active region.

During the second transit, the Moon fully occults the active region while uncovering relatively dimmer portions of the solar disk in the vicinity of the polar regions which results in the net effect of a dip.

During the third transit, at the beginning, the Moon partially occults the active region while uncovering the north-western off-limb and limb regions which are quite bright causing an increase in the profile. Then, the Moon further occults the active region almost fully which causes a decrease in the profile finally resulting in the net effect of a bump.

It can be deduced that for all the observed and simulated SWAVINT and AIA average intensity profiles, the background irradiance is also important with respect to the irradiance coming from the active region 11087. On the other hand, the LYRA short-wavelength channels are biased towards the active region 11087 as they observe SXR irradiance as well as EUV, and the way of occultation of the active region does not seem to matter for them. This deduction is the same as the one that we made in explaining the major differences between the SWAVINT and LYRA channel 2-4 variations in July 2010 in Section 3.1.

\section{Conclusions}

In this paper, we demonstrated the possibility of using the SWAP integrated flux as an additional radiometric channel complementary to the other solar monitoring instrument onboard PROBA2, viz. LYRA.

In order for SWAP to act as a radiometric channel, first, it should not degrade significantly during its mission. A detailed detector response analysis has been presented in [8] 

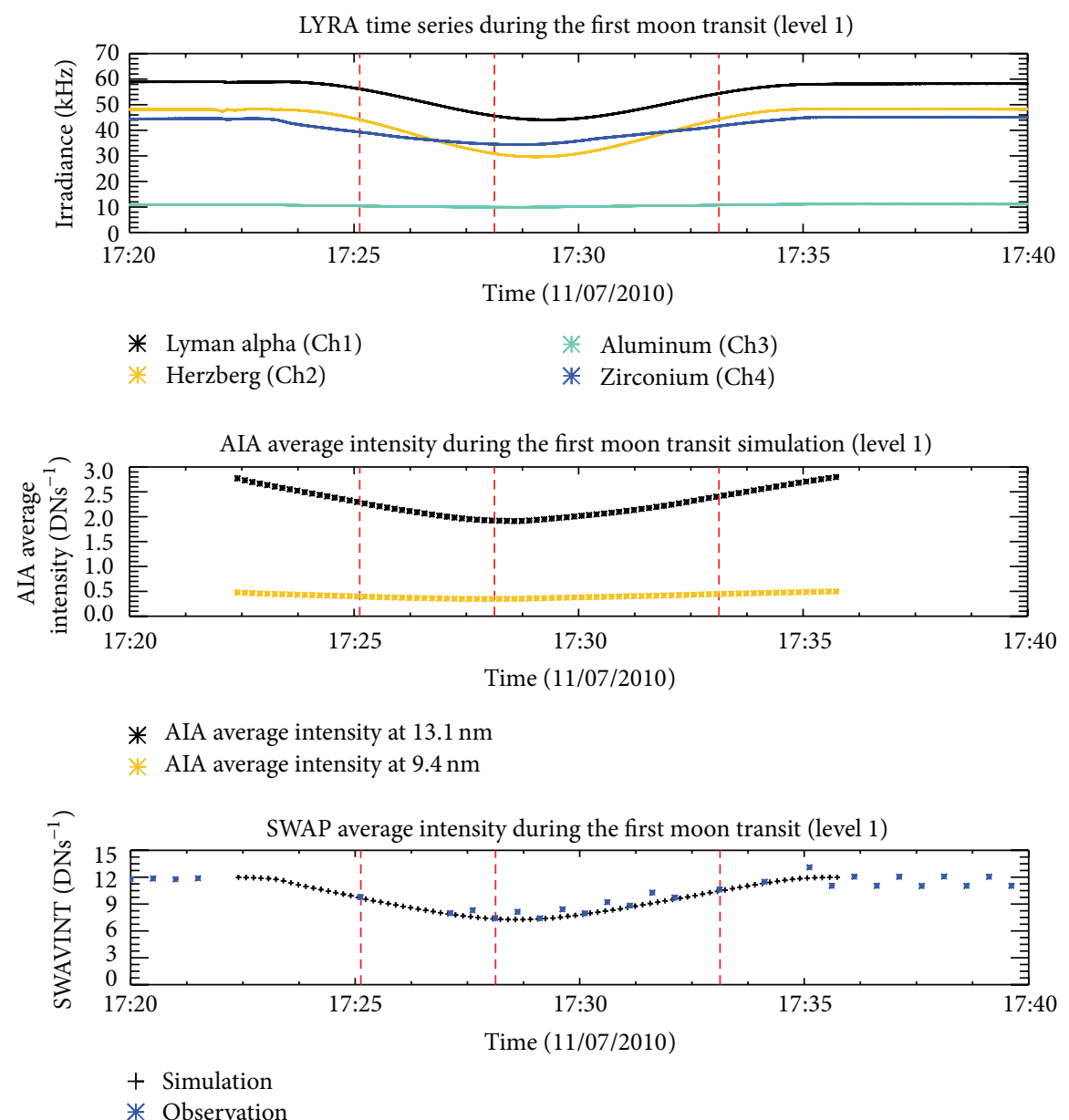

(a)

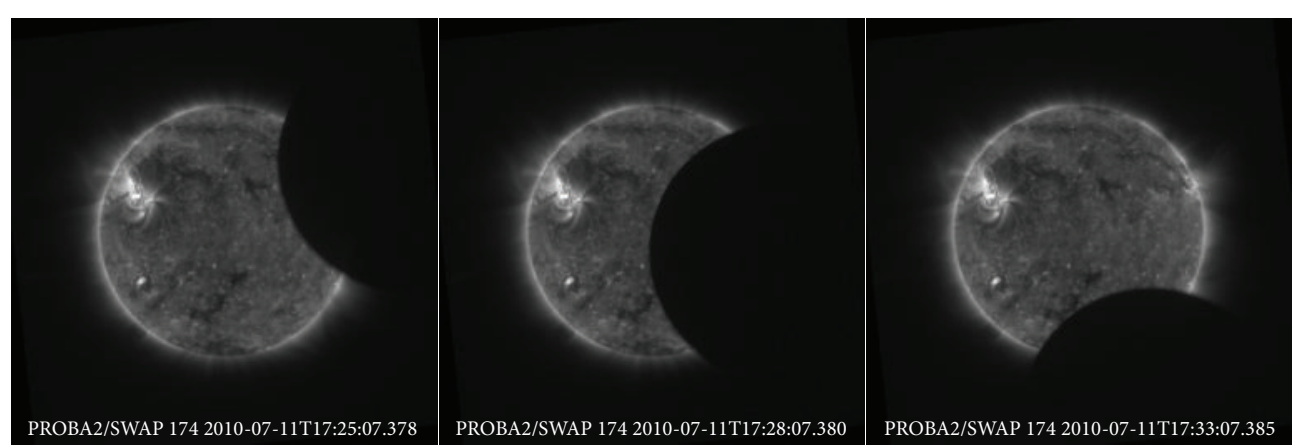

(b)

FIGURE 8: LYRA level 1 data measured by all four channels of unit 2, measured and simulated SWAVINT, and simulated AIA average intensity variations during the first transit of the Moon (a) together with certain SWAP images taken during the first transit of the Moon (b) whose times of observation are indicated in vertical lines on the graphs.

concluding that SWAP has not degraded significantly during roughly the first two years of its mission.

The long-term variability of the integrated flux of SWAP over the solar disk has shown a good correlation with LYRA level 3 channel 2-4 data and, in particular, an even better correlation with the ESP level 1 channel 18 data between 2010 and 2013. Furthermore, LYRA level 3 channel 2-4 data correlate well with the ESP level 1 data taken at combined responses of the channels QD and 18 between 2010 and 2013 and, in particular, in July 2010. Moreover, the ESP channel 18 data correlate well with SWAVINT in July 2010 as well. All these good correlations support the usage of SWAP as an additional radiometric channel.

We also focused on the SWAP and LYRA solar irradiance observations in July 2010 and explained the differences in terms of the coronal features (active regions and coronal 
LYRA time series during the second moon transit (level 1)
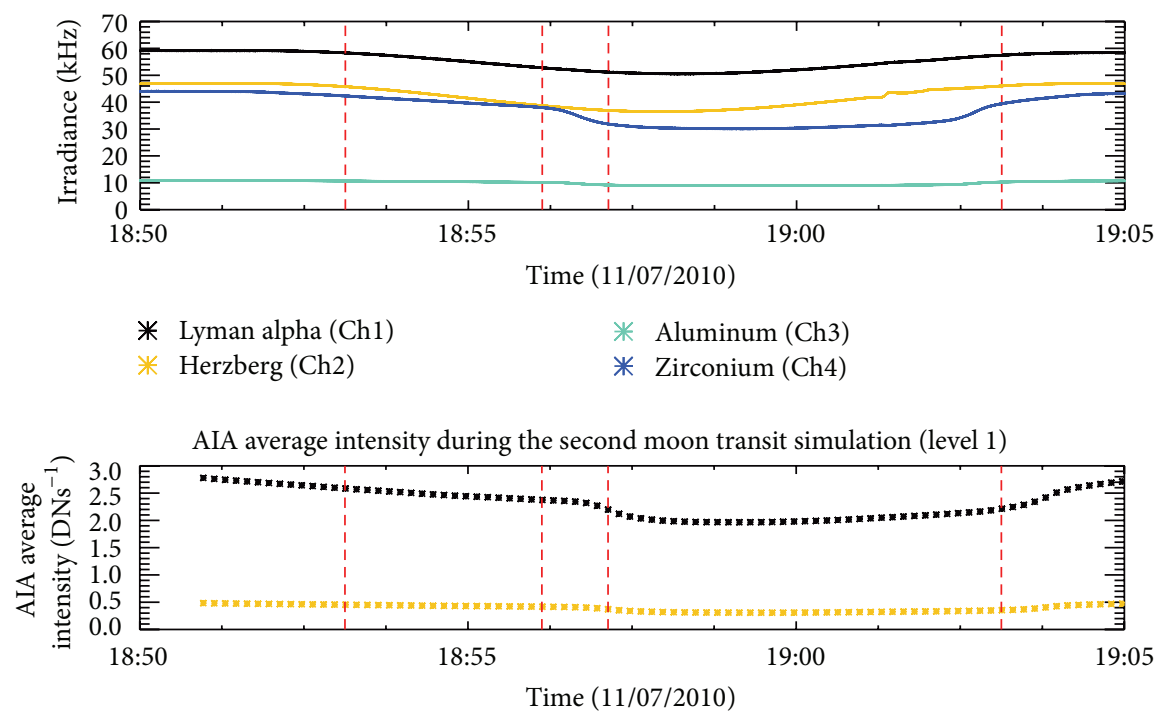

AIA average intensity at $13.1 \mathrm{~nm}$

* AIA average intensity at $9.4 \mathrm{~nm}$

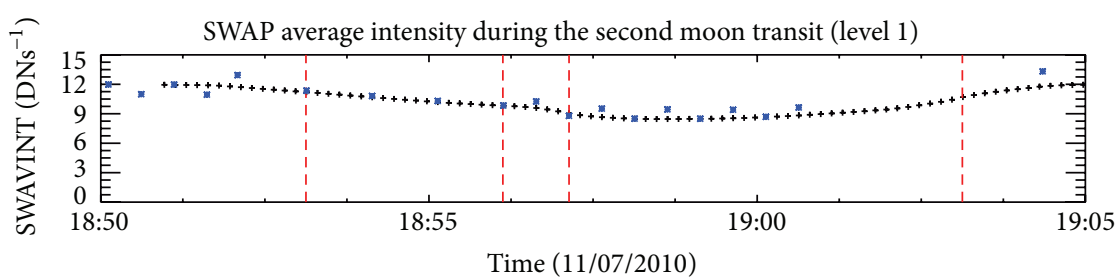

+ Simulation

* Observation

(a)

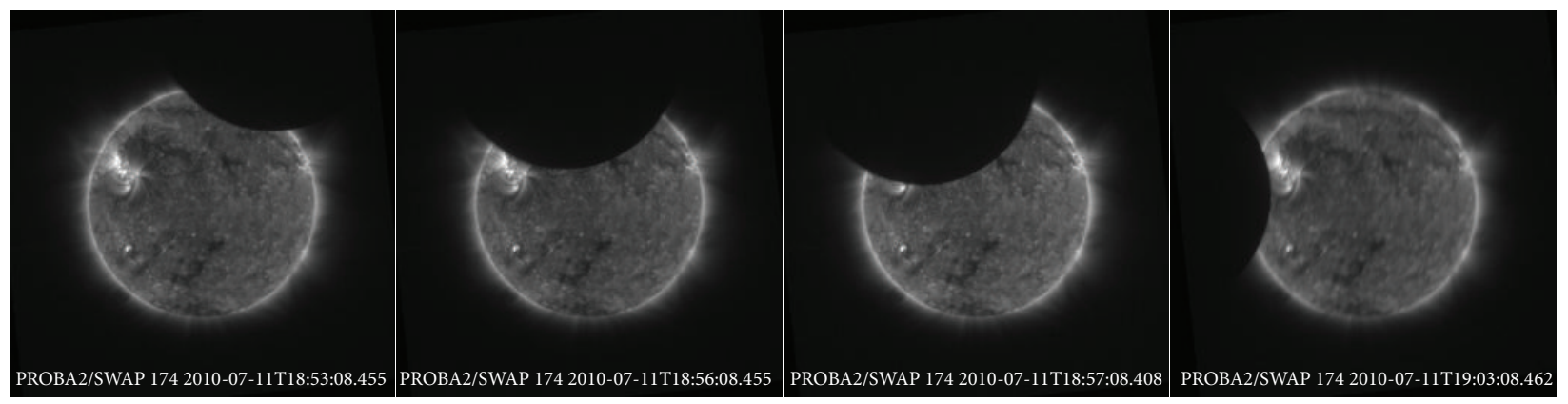

(b)

FIGURE 9: LYRA level 1 data measured by all four channels of unit 2, measured and simulated SWAVINT, and simulated AIA average intensity variations during the second transit of the Moon (a) together with certain SWAP images taken during the second transit of the Moon (b) whose times of observation are indicated in vertical lines on the graphs.

holes) visible on the solar disk. This interpretation was further supported by studying the July 11, 2010 solar eclipse in which an active region was temporarily occulted by the Moon.

Our results demonstrate that the SWAP integrated flux can indeed be used as an additional radiometric channel of LYRA yielding insight on which coronal features are contributing in the timelines. Furthermore, SWAP provides spatial information on coronal features and hence can explain why certain variations are seen in LYRA and where the source regions are located.

\section{Conflict of Interests}

The authors declare that there is no conflict of interests regarding the publication of this paper. 
LYRA time series during the third moon transit (level 1)
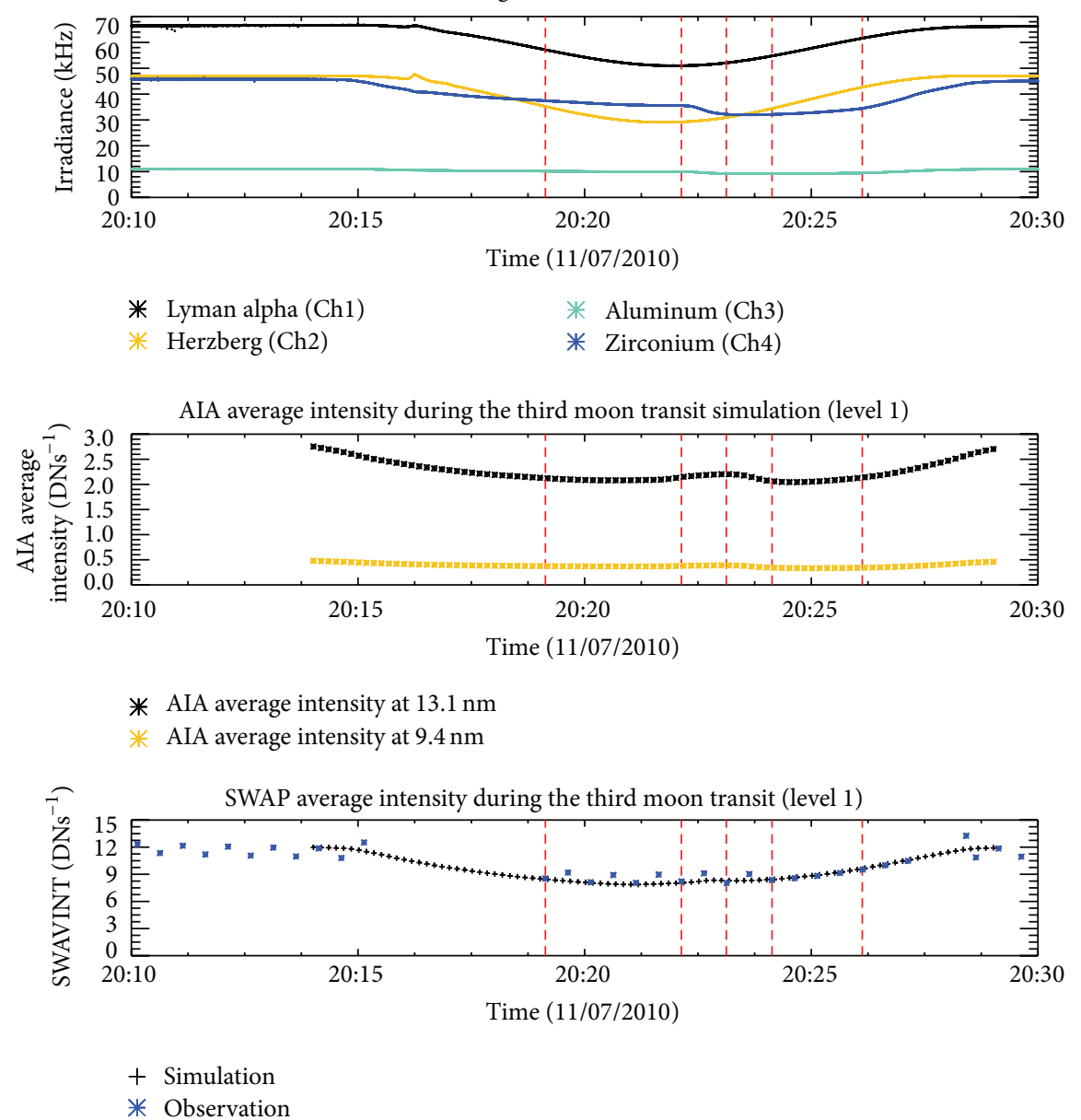

(a)

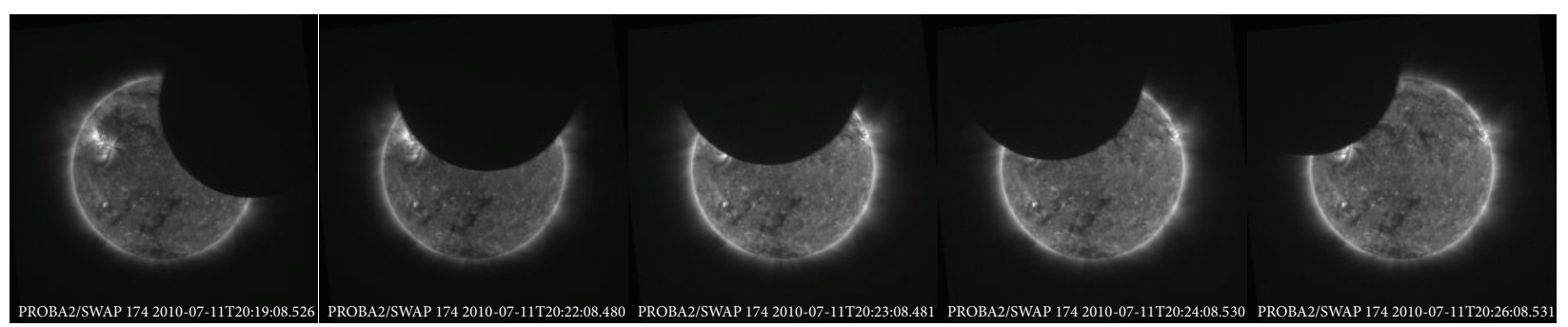

(b)

FIGURE 10: LYRA level 1 data measured by all four channels of unit 2, measured and simulated SWAVINT, and simulated AIA average intensity variations during the third transit of the Moon (a) together with certain SWAP images taken during the third transit of the Moon (b) whose times of observation are indicated in vertical lines on the graphs.

\section{Acknowledgments}

The authors acknowledge the support from the Belgian Federal Science Policy Office (BELSPO) through the ESAPRODEX 9 project entitled "SIDC Data Exploitation." SWAP has been developed and tested by the Centre Spatial de Liège (CSL) of the University of Liège, and calibrated in collaboration with the Royal Observatory of Belgium (ROB), within the framework of a European collaboration and with the support of the Belgian industry, in particular QinetiQ
Space NV. LYRA is a project of CSL, the PhysikalischMeteorologisches Observatorium Davos (PMOD), and ROB funded by BELSPO and the Swiss Bundesamt für Bildung und Wissenschaft. The authors appreciate the availability of the NASA solar data from the highly successful SDO mission and the PROBA2 data from the P2SC. The authors thank Dr. David Berghmans, Ms. Marie Dominique, and Mr. Ingolf Dammasch from ROB and Dr. Anik De Groof from ESA for their useful comments and suggestions. 


\section{References}

[1] F. Teston, R. Creasey, J. Bermyn et al., "PROBA: ESA's autonomy and technology demonstration mission," in Proceedings of the 13th Annual AIAA/USU Conference on Small Satellites, Logan, Utah, USA, 1999.

[2] P. Rochus, J. M. Defise, J. H. Lecat et al., "PROBA II payload: a Belgian mini space weather observatory," in Proceedings of the 55th International Astronautical Congress, vol. 5, pp. 3403-3412, Vancouver, Canada, October 2004.

[3] S. Santandrea, K. Gantois, K. Strauch et al., "PROBA2: mission and spacecraft overview," Solar Physics, vol. 286, no. 1, pp. 5-19, 2013.

[4] M. Dominique, J. F. Hochedez, W. Schmutz et al., "The LYRA instrument onboard PROBA2: description and in-flight performance," Solar Physics, vol. 286, no. 1, pp. 21-42, 2013.

[5] K. Gantois, F. Teston, O. Montenbruck et al., "PROBA-2: mission and new technologies overview," in Proceedings of the 4S Symposium: Small Satellites, Systems and Services, D. Danesy, Ed., Chia, Italy, September 2006.

[6] D. Berghmans, J. F. Hochedez, J. M. Defise et al., "SWAP onboard PROBA 2, a new EUV imager for solar monitoring," Advances in Space Research, vol. 38, no. 8, pp. 1807-1811, 2006.

[7] J.-P. Halain, D. Berghmans, J.-M. Defise et al., "First light of SWAP on-board PROBA2," in Space Telescopes and Instrumentation 2010: Ultraviolet to Gamma Ray, vol. 7732 of Proceedings of SPIE, San Diego, Calif, USA, July 2010.

[8] J. P. Halain, D. Berghmans, D. B. Seaton et al., "The SWAP EUV imaging telescope. Part II: in-flight performance and calibration," Solar Physics, vol. 286, no. 1, pp. 67-91, 2013.

[9] D. B. Seaton, D. Berghmans, B. Nicula et al., "The SWAP EUV imaging telescope part I: instrument overview and pre-flight testing," Solar Physics, vol. 286, no. 1, pp. 43-65, 2013.

[10] J.-P. Delaboudinière, G. E. Artzner, J. Brunaud et al., "EIT: Extreme-ultraviolet Imaging Telescope for the SOHO mission," Solar Physics, vol. 162, no. 1-2, pp. 291-312, 1995.

[11] J. R. Lemen, A. M. Title, D. J. Akin et al., "The Atmospheric Imaging Assembly (AIA) on the Solar Dynamics Observatory (SDO)," Solar Physics, vol. 275, no. 1-2, pp. 17-40, 2012.

[12] J.-F. Hochedez, W. Schmutz, Y. Stockman et al., "LYRA, a solar UV radiometer on Proba2," Advances in Space Research, vol. 37, no. 2, pp. 303-312, 2006.

[13] A. Ben Moussa, I. E. Dammasch, J.-F. Hochedez et al., "Preflight calibration of LYRA, the solar VUV radiometer on board PROBA2," Astronomy \& Astrophysics, vol. 508, no. 2, pp. 10851094, 2009.

[14] S. T. Kumara, R. Kariyappa, M. Dominique et al., "Preliminary results on irradiance measurements from lyra and swap," Advances in Astronomy, vol. 2012, Article ID 623709, 5 pages, 2012.

[15] J. Zender, D. Berghmans, D. S. Bloomfield et al., "The Projects for Onboard Autonomy (PROBA2) science centre: Sun Watcher Using APS Detectors and Image Processing (SWAP) and LargeYield Radiometer (LYRA) science operations and data products," Solar Physics, vol. 286, no. 1, pp. 93-110, 2013. 

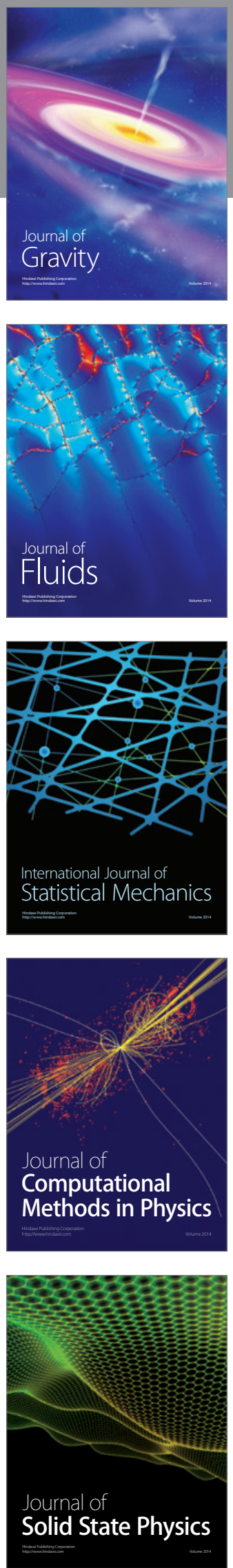

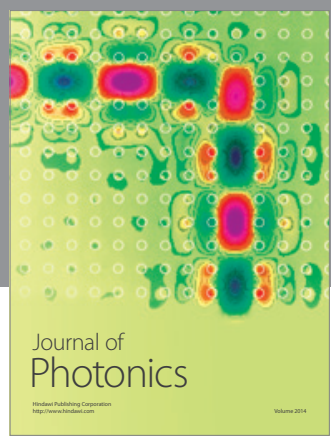

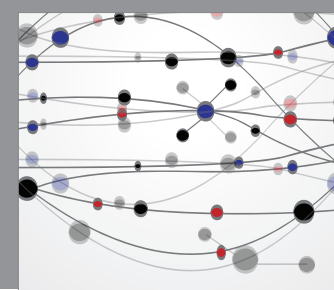

The Scientific World Journal

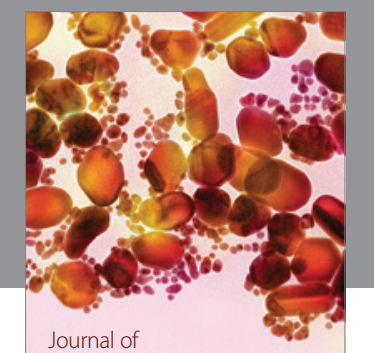

Soft Matter
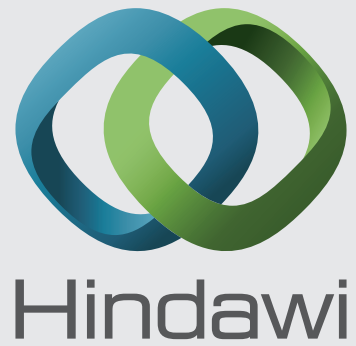

Submit your manuscripts at

http://www.hindawi.com
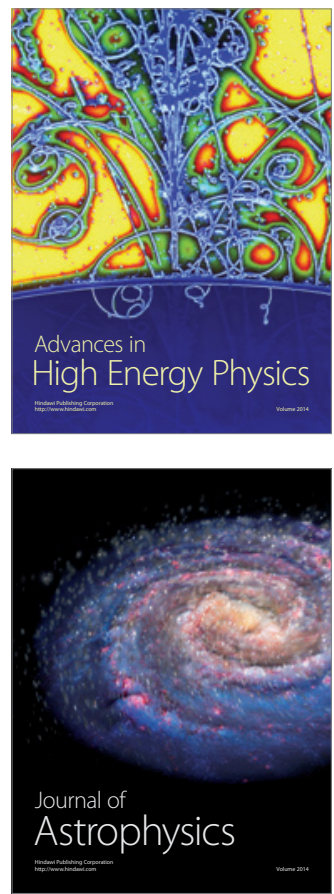
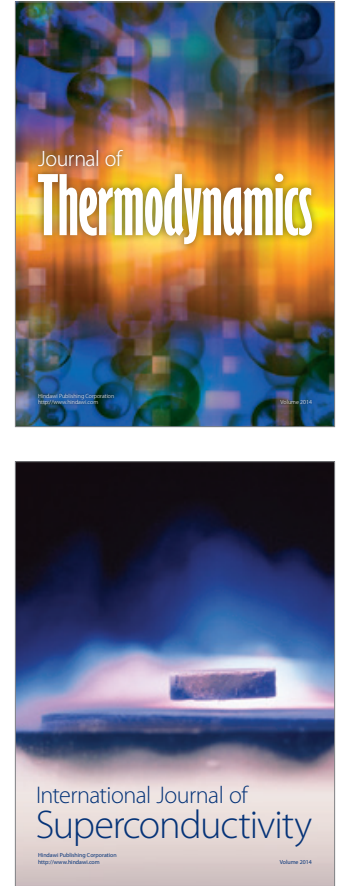
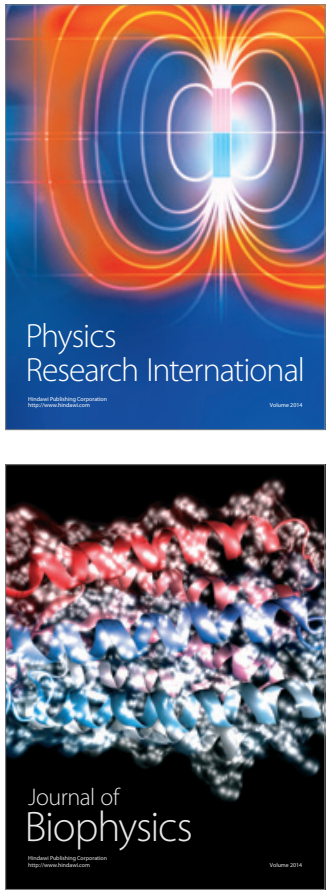
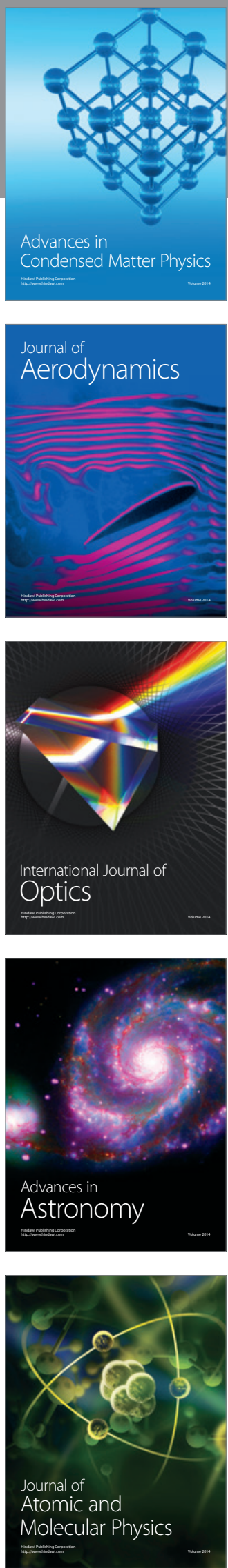